Max-Planck-Institut für Kohlenforschung

Journal article published in: J. Chem. Theory Comput. 2016, 12, 8, 3561-3570

https://pubs.acs.org/doi/abs/10.1021/acs.jctc.6b00269

This article may be used for non-commercial purposes.

\title{
Periodic boundary conditions in QM/MM calculations: Implementation and tests
}

\author{
Tatiana Vasilevskaya and Walter Thiel* \\ Max-Planck-Institut für Kohlenforschung, Mülheim an der Ruhr, Germany \\ E-mail: thiel@kofo.mpg.de
}

\begin{abstract}
Quantum mechanics/molecular mechanics (QM/MM) simulations of reactions in solutions and in solvated enzymes can be performed using the QM/MM-Ewald approach with periodic boundary conditions $(\mathrm{PBC})$ or a non-periodic treatment with a finite solvent shell (droplet model). To avoid the changes in QM codes that are required in standard QM/MM-Ewald implementations, we present a general method (Gen-Ew) for periodic QM/MM calculations that can be used with any QM method in the QM/MM framework. The Gen-Ew approach approximates the QM/MM-Ewald method by representing the $\mathrm{PBC}$ potential by virtual charges on a sphere and the QM density by electrostatic potential (ESP) charges. Test calculations show that the deviations between Gen-Ew and QM/MM-Ewald results are generally small enough to justify the application of the Gen-Ew method in the absence of a suitable QM/MM-Ewald implementation. We compare the results from periodic QM/MM calculations (QM/MM-Ewald, Gen-Ew) to their non-periodic counterparts (droplet model) for five test reactions in water and for the Claisen rearrangement in chorismate mutase. The periodic and non-periodic QM/MM treatments give similar free energy profiles for the reactions in solution (umbrella sampling,
\end{abstract}

*To whom correspondence should be addressed 
free energy deviations of the order of $1 \mathrm{kcal} / \mathrm{mol}$ ) and essentially the same energy profile (constrained geometry optimizations) for the Claisen rearrangement in chorismate mutase. In all cases considered, long-range electrostatic interactions are thus well captured by non-periodic QM/MM calculations in a water droplet of reasonable size (radius of 15-20 $\mathrm{A}$ ). This provides further justification for the wide-spread use of the computationally efficient droplet model in QM/MM studies of reactions in solution and in enzymes. 


\section{Introduction}

Solvent effects on chemical reactions have been extensively studied both experimentally and computationally. ${ }^{[-5]}$ In many cases, reactions are strongly affected by the solvent, for example in the Claisen rearrangement $\mathrm{t}^{2 / 3 / 6+9}$ or in Diels-Alder reactions. ${ }^{2 / 3|10| 11}$ The solvent environment may also change the reaction mechanism by stabilizing or destabilizing particular structures; for example, the glycine zwitterion does not exist in the gas phase ${ }^{12 \mid 13}$ but dominates in aqueous media.

Solvent effects must clearly be taken into account in realistic simulations of condensed-phase reactions. Well suited for this purpose are combined quantum mechanics/molecular mechanics (QM/MM) approaches, 14 in which the solute is treated quantum mechanically and the solvent by classical force fields. Solvent effects often arise from solvent-solute electrostatic interactions: short-range interactions will normally be dominant, but long-range electrostatics may also play an important role. Long-range electrostatic interactions are also known to influence the structural properties of biomolecules, for example of proteins $\frac{15}{15}$ and nucleic acids. $\underline{16[17}$

Several computational methods are available to treat long-range electrostatics in a QM/MM framework using periodic boundary conditions (PBC). The first published QM/MM-Ewald method is designed for application with NDDO-based semiempirical methods: the QM charge density is modeled by Mulliken charges and the PBC contributions are represented by additional terms that are added to the Fock matrix. $\frac{18}{18}$ A faster implementation of this approach makes use of the particle mesh Ewald (PME) algorithm. ${ }^{19 \mid 20}$ There are also implementations for QM/MM methods that employ density functional theory (DFT) as QM component. ${ }^{21 / 22}$ All these approaches require modifications of the underlying QM programs and are thus limited to particular QM methods. A recent general QM/MM implementation for two- and three-dimensional systems relies on mechanical embedding and thus treats long-range electrostatics at the MM level. $\stackrel{23}{2}$ The original QM/MM-Ewald method can also be applied in combination with Hartree-Fock (HF) and DFT methods if minimal basis sets are employed, but the use of Mulliken charges causes instabilities for extended basis sets; these problems are overcome by representing the QM charge density by "ChElPG" atomic charges using atom-centered Lebedev grids, which enables QM/MM-Ewald MD simulations with 
HF, DFT, and post-HF QM treatments. ${ }^{24 \mid 25}$ In a recently proposed multiscale method to perform ab initio QM/MM calculations with periodic boundary conditions, the total energy of the system is evaluated using the original QM/MM-Ewald method with a semiempirical QM treatment and then corrected on the fly at the ab initio level of theory within the real space cutoff; in this approach, a multiple time step technique is applied to minimize the number of costly ab initio calculations and thus to speed up the QM/MM MD simulations. 26

In this article, we first present another generalized QM/MM-Ewald method that can be used with any QM program and QM method. Thereafter we compare periodic and non-periodic QM/MM calculations on several test reactions and analyze the influence of long-range electrostatics on energy and free energy profiles. The comparisons cover the original 18 and our generalized QM/MMEwald approach as well as a non-periodic treatment using the standard QM/MM droplet model with a finite solvent sphere. 14

\section{Methods}

\subsection{QM/MM-Ewald method}

We start with a brief overview over the original QM/MM-Ewald implementation for semiempirical QM methods $\frac{18}{18}$ to establish notation. The electrostatic energy of a periodic QM/MM system $E_{Q M / M M}^{P B C}$ is defined as the sum of non-periodic real-space energy contributions $\left(E^{R S}\right), \mathrm{PBC}$ correction terms $\left(\triangle E^{P B C}\right)$, and $\mathrm{PBC}$ contributions from the $\mathrm{MM}$ region $\left(E_{M M}^{P B C}\right)$ :

$$
E_{Q M / M M}^{P B C}=E_{Q M}^{R S}[\rho, \rho]+\Delta E_{Q M-Q M}^{P B C}[\rho, \rho]+E_{Q M-M M}^{R S}[\rho, q]+\Delta E_{Q M-M M}^{P B C}[\rho, q]+E_{M M}^{P B C}[q, q]
$$

where $\rho$ represents the electron density and the core charges of the QM atoms, and $q$ denotes the point charges of the $\mathrm{MM}$ atoms. The real-space energy terms $E_{Q M}^{R S}$ and $E_{Q M-M M}^{R S}$ are obtained from a standard QM/MM calculation on a system obeying the minimum image convention, i.e. with dimensions that are equal to or less than half of the unit cell box length used in the PBC 
calculation. For the PBC correction terms $\triangle E_{Q M-Q M}^{P B C}$ and $\triangle E_{Q M-M M}^{P B C}$ it is assumed that the QM charge density can be approximated with Mulliken charges $Q$ :

$$
E_{Q M / M M}^{P B C} \approx E_{Q M}^{R S}[\rho, \rho]+\Delta E_{Q M-Q M}^{P B C}[Q, Q]+E_{Q M-M M}^{R S}[\rho, q]+\Delta E_{Q M-M M}^{P B C}[Q, q]+E_{M M}^{P B C}[q, q]
$$

Following previous notation, $\frac{18}{,}$ we label QM atoms by $\alpha$ and $\beta$, MM atoms by $i$ and $j$, and distance vectors between atoms are denoted by $\mathbf{R}$ (with suitable indices identifying the two atoms). If the Ewald parameter $\kappa$ is chosen according to the minimum image convention, the PBC correction terms can be written as

$$
\begin{gathered}
\Delta E_{Q M-Q M}^{P B C}[Q, Q]=\frac{1}{2} \sum_{\alpha}^{N_{Q M}} \sum_{\beta}^{N_{Q M}} Q_{\alpha} Q_{\beta} \Delta \psi\left(\mathbf{R}_{\alpha \beta}\right) \\
\Delta E_{Q M-M M}^{P B C}[Q, q]=\sum_{\alpha}^{N_{Q M}} Q_{\alpha} \sum_{j}^{N_{M M}} q_{j} \Delta \psi\left(\mathbf{R}_{\alpha j}\right)
\end{gathered}
$$

where the so-called ${ }^{18}$ Ewald pair potential $\Delta \psi\left(\mathbf{R}_{\alpha \beta}\right)$ is

$$
\Delta \psi\left(\mathbf{R}_{\alpha \beta}\right)=\frac{4 \pi}{V} \sum_{|k| \neq 0} \frac{\exp \left(-k^{2} / 4 \kappa^{2}\right)}{k^{2}} \cdot \cos \left(\mathbf{k} \cdot \mathbf{R}_{\alpha \beta}\right)-\frac{\operatorname{erf}\left(\kappa\left|\mathbf{R}_{\alpha \beta}\right|\right)}{\left|\mathbf{R}_{\alpha \beta}\right|}
$$

Here, $V$ is the volume of the unit cell, $\mathbf{k}$ is the lattice vector of the reciprocal lattice, and $\operatorname{erf}(x)$ is the error function. The PBC contributions are taken into account during the self-consistent field (SCF) iterations by adding the following terms to the diagonal Fock matrix elements $F_{\mu \mu}$ for basis functions $\mu$ at QM atom $\alpha$ :

$$
\Delta F_{\mu \mu}^{Q M / M M-E w a l d}=-\left(\sum_{\beta}^{N_{Q M}} Q_{\beta} \Delta \psi\left(\mathbf{R}_{\alpha \beta}\right)+\sum_{j}^{N_{M M}} q_{j} \Delta \psi\left(\mathbf{R}_{\alpha j}\right)\right)
$$

The QM-QM PBC correction to the Fock matrix (first term in eq. 6) must be recalculated at each SCF iteration, while the QM-MM PBC correction (second term in eq. 6) is constant since the MM charges do not change. 
The PBC corrections affect the total energy and the forces on the atoms. Their contribution to the gradient can be evaluated by taking the first derivative of $\triangle E_{Q M-Q M}^{P B C}$ and $\triangle E_{Q M-M M}^{P B C}$ with respect to the atomic coordinates. For a given $\mathrm{QM}$ atom $A$ at position $\mathbf{R}_{A}$ this yields formally:

$$
\begin{gathered}
\frac{\partial}{\partial \mathbf{R}_{A}} \Delta E_{Q M-Q M}^{P B C}=Q_{A} \sum_{\beta}^{N_{Q M}} Q_{\beta} \frac{\partial}{\partial \mathbf{R}_{A}} \Delta \psi\left(\mathbf{R}_{A \beta}\right)+\sum_{\alpha}^{N_{Q M}} \frac{\partial Q_{\alpha}}{\partial \mathbf{R}_{A}} \sum_{\beta}^{N_{Q M}} Q_{\beta} \Delta \psi\left(\mathbf{R}_{\alpha \beta}\right) \\
\frac{\partial}{\partial \mathbf{R}_{A}} \Delta E_{Q M-M M}^{P B C}=Q_{A} \sum_{j}^{N_{M M}} q_{j} \frac{\partial}{\partial \mathbf{R}_{A}} \Delta \psi\left(\mathbf{R}_{A j}\right)+\sum_{\alpha}^{N_{Q M}} \frac{\partial Q_{\alpha}}{\partial \mathbf{R}_{A}} \sum_{j}^{N_{M M}} q_{j} \Delta \psi\left(\mathbf{R}_{\alpha j}\right)
\end{gathered}
$$

The orbital coefficients, and hence also the Mulliken charges, are variationally optimized in the SCF procedure. Therefore the Mulliken charge derivatives, $\partial Q_{\alpha} / \partial \mathbf{R}_{A}$, are zero at the SCF level. This results in simplified working equations for the gradient components on the QM atoms (which hold only approximately when using other types of QM charges, see below):

$$
\begin{gathered}
\frac{\partial}{\partial \mathbf{R}_{A}} \Delta E_{Q M-Q M}^{P B C}=Q_{A} \sum_{\beta}^{N_{Q M}} Q_{\beta} \frac{\partial}{\partial \mathbf{R}_{A}} \Delta \psi\left(\mathbf{R}_{A \beta}\right) \\
\frac{\partial}{\partial \mathbf{R}_{A}} \Delta E_{Q M-M M}^{P B C}=Q_{A} \sum_{j}^{N_{M M}} q_{j} \frac{\partial}{\partial \mathbf{R}_{A}} \Delta \psi\left(\mathbf{R}_{A j}\right)
\end{gathered}
$$

The gradient components on a given MM atom $I$ at position $\mathbf{R}_{I}$ are calculated according to

$$
\frac{\partial}{\partial \mathbf{R}_{I}} \Delta E_{Q M-M M}^{P B C}=q_{I} \sum_{\beta}^{N_{Q M}} Q_{\beta} \frac{\partial}{\partial \mathbf{R}_{I}} \Delta \psi\left(\mathbf{R}_{I \beta}\right)
$$

Our implementation of the original QM/MM-Ewald method ${ }^{18}$ evaluates the PBC corrections to the energy, the Fock matrix, and the gradient by using eqs. 3.5, eq. 6, and eqs. 9.11, respectively.

\subsection{Generalized QM/MM-Ewald method}

The main objectives in the development of the generalized QM/MM-Ewald (Gen-Ew) method are efficiency and flexibility, i.e. it should work with any kind of QM method in QM/MM approaches. 
For this purpose, the theoretical framework of the original QM/MM-Ewald method is retained but approximations are introduced in the evaluation of the QM/MM PBC correction terms. Motivated by the concepts underlying the solvated macromolecule boundary potential (SMBP) approach, 27 we model the QM density by electrostatic potential (ESP) charges $Q_{\alpha}^{E S P}$ at the QM atoms $\alpha$ and evaluate their interaction with the PBC potential $\varphi^{P B C}\left(\mathbf{R}_{\alpha}\right)$ to determine the PBC energy corrections:

$$
\begin{gathered}
\Delta E_{Q M-Q M}^{P B C}+\Delta E_{Q M-M M}^{P B C}=\sum_{\alpha}^{N_{Q M}} Q_{\alpha}^{E S P} \varphi^{P B C}\left(\mathbf{R}_{\alpha}\right) \\
\varphi^{P B C}\left(\mathbf{R}_{\alpha}\right)=\frac{1}{2} \sum_{\beta}^{N_{Q M}} Q_{\beta}^{E S P} \Delta \psi\left(\mathbf{R}_{\alpha \beta}\right)+\sum_{j}^{N_{M M}} q_{j} \Delta \psi\left(\mathbf{R}_{\alpha j}\right)
\end{gathered}
$$

The PBC potential is projected onto a set of $N_{\text {virt }}$ virtual charges $\left\{q_{m}^{\text {virt }}\right\}$ at positions $\mathbf{R}_{m}$, which are uniformly distributed on a sphere centered at the geometrical center of the QM part (Figure 1).

$$
\varphi_{\text {approx }}^{P B C}\left(\mathbf{R}_{\alpha}\right) \approx \sum_{m}^{N_{\text {virt }}} \frac{q_{m}^{\text {virt }}}{\left|\mathbf{R}_{\alpha}-\mathbf{R}_{m}\right|}
$$




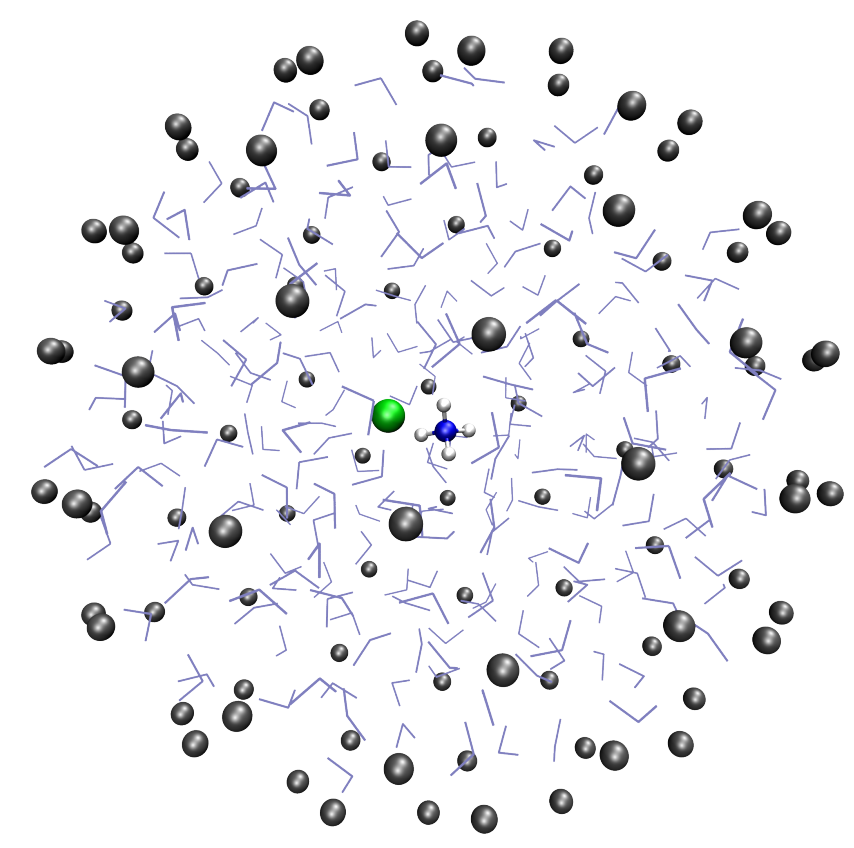

Figure 1: Sphere charges (dark gray) used to represent the PBC potential in the case of ammonium chloride solvated in water. The solute (QM region) is shown in a ball-and-stick model, while the solvent molecules (MM region) are indicated as blue lines.

The virtual charges are optimized such that they reproduce the PBC potential $\varphi^{P B C}\left(\mathbf{R}_{\alpha}\right)$ (eq. 13 at the positions of the QM atoms. As in previous work, 27 this is achieved by minimizing the penalty function

$$
\tilde{f}=\sum_{\alpha}^{N_{Q M}}\left(\varphi^{P B C}\left(\mathbf{R}_{\alpha}\right)-\sum_{m}^{N_{v i r t}} \frac{q_{m}^{\text {virt }}}{\left|\mathbf{R}_{\alpha}-\mathbf{R}_{m}\right|}\right)^{2}
$$

using the conjugate gradient algorithm. The minimization starts with the virtual charges set to zero and finishes when $\varphi^{P B C}$ is reproduced with an absolute deviation of less than $2.0 \cdot 10^{-5}$ au at the position of every QM atom.

The iterative minimization proceeds as follows. (1) The QM ESP charges are taken from the previous step. In the very first step, all QM atom charges are assumed to be zero. (2) The PBC potential $\varphi^{P B C}$ is calculated at the position of each $\mathrm{QM}$ atom. (3) The PBC potential $\varphi^{P B C}$ is projected on a set of virtual charges. (4) The QM wave function is computed in the field of the virtual charges and the MM point charges; upon SCF convergence, new QM ESP charges are calculated. (5) The QM ESP charges are checked for convergence; unless convergence is achieved, 
the algorithm returns to step 2. (6) Upon convergence in step 5, the force contributions from the PBC potential are computed and added to the gradient. In this procedure, real-space cutoffs for the QM-MM electrostic interactions may be introduced in steps 2 and 4.

In the original QM/MM-Ewald method, the $\mathrm{PBC}$ contributions give rise to extra terms in the Fock matrix (see above, eq. 6). These contributions are handled in a formally different manner in the Gen-Ew method: virtual charges representing the PBC potential $\varphi^{P B C}$ at the position of QM atoms are added and incorporated into the core Hamiltonian and the Fock matrix as extra one-electron terms.

$$
\hat{H}^{G e n-E w}=\hat{F}^{G e n-E w}=\sum_{\alpha}^{N_{Q M}} \sum_{m}^{N_{v i r t}} \frac{Z_{\alpha} q_{m}^{\text {virt }}}{\left|\mathbf{R}_{\alpha}-\mathbf{R}_{m}\right|}-\sum_{v}^{N_{e l}} \sum_{m}^{N_{v i r t}} \frac{q_{m}^{\text {virt }}}{\left|\mathbf{r}_{v}-\mathbf{R}_{m}\right|}
$$

The two terms describe the interactions of the virtual charges with the core charges $Z_{\alpha}$ of the QM atoms and the electrons ( $v)$ of the QM region, respectively. We note that the corrections $\Delta F_{\mu \mu}^{Q M / M M-E w a l d}$ are the same for all basis functions $\mu$ at QM atom $\alpha$ (eq. 6), whereas the corresponding Gen-Ew corrections are evaluated as matrix elements of $\hat{F}^{G e n-E w}$ and may thus differ for different basis functions at a given QM atom (eq. 16).

The PBC contributions to the gradient are the derivatives of the $\mathrm{PBC}$ energy correction (eq. 12) with respect to the atomic coordinates. For the QM atoms the differentiation can take advantage of the virtual-charge representation of the PBC potential (eq. 14) which leads to a simple point-charge expression in terms of ESP and virtual charges. In the case of the MM atoms, the differentiation of eqs. 12,13 yields a formula completely analogous to eq. 11, except that the Mulliken charges are replaced by ESP charges. All PBC contributions to the gradient are thus easily available in the Gen-Ew method.

One obvious issue is how well the QM/MM-Ewald results can be reproduced by their GenEw counterparts. Clearly the Mulliken and ESP charges will differ to some extent, and the PBC potential is mimicked by the virtual charges only approximately (albeit quite accurately). Hence, there will be differences in the PBC energy corrections as well as in the corresponding gradient contributions. We note, in particular, that the gradient evaluation in the Gen-Ew method neglects 
the terms involving charge derivatives (eqs. 74, which vanish for Mulliken charges but not for ESP charges (see above). These accuracy issues will be addressed in the following sections through numerical tests.

\section{Test systems}

The effects of long-range electrostatics should be particularly pronounced for reactions of charged species in water. Therefore we chose to study the dissociation of salts $\left(\mathrm{NH}_{4} \mathrm{Cl} \text { and } \mathrm{NH}_{4} \mathrm{PO}_{3}\right)^{\frac{18}{18}}$ as well as the bimolecular nucleophilic substitution $\left(\mathrm{S}_{\mathrm{N}} 2\right)$ reaction between chloride and chloromethane in aqueous solution. 28 As an example of a reaction of neutral species, we investigated the Claisen rearrangement of allylvinyl ether in water. $\frac{32-36}{36}$ To explore the influence of long-range electrostatics on the structure of biomolecules, we studied the internal rotation of glycine dipeptide (Ace-Gly-NMe) around one of the backbone dihedral angles $(\varphi)$, again in water. $\frac{37 / 38}{}$ For these five test systems we compared potential of mean force (PMF) profiles obtained from periodic simulations (QM/MM-Ewald and Gen-Ew methods) and from non-periodic simulations (droplet model). We also carried out periodic and non-periodic MD simulations for glycine in water to check for energy conservation in the NVE ensemble. Finally, we performed a series of constrained geometry optimizations to compute a potential energy profile of the Claisen rearrangement in the enzyme chorismate mutase (conversion of chorismate to prephenate). $39-60$

In the QM/MM calculations, the QM region consisted of the solute (simulations in aqueous solution) or the substrate (enzymatic reaction). The semiempirical AM1 Hamiltonian ${ }^{61}$ was used for ammonium chloride and glycine dipeptide; PM3 ${ }^{62}$ for glycine; MNDO/ $\mathrm{d}^{63}$ for ammonium metaphosphate; and PM3-PDDG ${ }^{64 \mid 65}$ for the Claisen rearrangement of allylvinyl ether, the $\mathrm{S}_{\mathrm{N}} 2$ reaction, and chorismate. The MM region consisted of the solvent (water) and included the protein environment in the case of the enzymatic reaction (chorismate mutase). MM water was always represented by the TIP3P model, 66 while chorismate mutase was described by the CHARMM force field. 67 


\section{Computational details}

The original QM/MM-Ewald method and the Gen-Ew method were implemented in a developmental version of the program package ChemShell. ${ }^{68 \mid 69}$ The smooth particle mesh Ewald (SPME) method $^{70}$ was also implemented in ChemShell to speed up the time-consuming reciprocal space calculations. The QM/MM calculations were performed using the MNDO program ${ }^{71}$ for the semiempirical QM calculations and the DL_POLY program ${ }^{72}$ for the MM calculations. Prior to QM/MM calculations all systems were equilibrated by means of a 500 ps classical NPT molecular dynamics simulation.

Table 1: Computational setup for the periodic calculations.

\begin{tabular}{l|c|c|c|c|c}
\hline System & $N$ & box size, $\AA$ & $r_{c u t}, \AA$ & $r_{c u t}^{\text {vdW }}, \AA$ & $\kappa, \AA^{-1}$ \\
\hline $\mathrm{NH}_{4} \mathrm{Cl}$ & 6861 & $41 \times 40 \times 41$ & 10.0 & 11.5 & 0.34 \\
$\mathrm{NH}_{4} \mathrm{PO}_{3}$ & 7173 & $40 \times 40 \times 42$ & 10.0 & 11.5 & 0.34 \\
$\mathrm{~S}_{\mathrm{N}} 2$ & 7797 & $42 \times 41 \times 43$ & 11.5 & 11.5 & 0.30 \\
allylvinyl ether & 3662 & $34 \times 33 \times 32$ & 15.5 & 15.5 & 0.24 \\
Ace-Gly-NMe & 8563 & $41 \times 46 \times 44$ & 15.5 & 15.5 & 0.24 \\
glycine & 7312 & $42 \times 41 \times 41$ & 10.5 & 11.5 & 0.32 \\
\hline
\end{tabular}

Table 2: Size of systems in the non-periodic calculations.

\begin{tabular}{l|c|c}
\hline System & $N$ & droplet radius, $\stackrel{\circ}{ }$ \\
\hline & 3627 & 20 \\
$\mathrm{NH}_{4} \mathrm{Cl}$ & 1890 & 15 \\
& 1023 & 12 \\
\hline $\mathrm{NH}_{4} \mathrm{PO}_{3}$ & 3675 & 20 \\
\hline $\mathrm{S}_{\mathrm{N}} 2$ & 4971 & 22 \\
\hline \multirow{2}{*}{ allylvinyl ether } & 3662 & 20 \\
& 1886 & 15 \\
\hline Ace-Gly-NMe & 3610 & 20 \\
\hline glycine & 12766 & 30 \\
\hline
\end{tabular}

Specific choices for the individual test systems are shown in Table 1 and Table 2. For each test system, the setup for the periodic MD simulations is specified in Table 1 in terms of the total number of atoms $N$, the box size, the cut-offs for the real-space electrostatics $\left(r_{c u t}\right)$ and the vander-Waals (vdW) interactions $\left(r_{c u t}^{v d W}\right)$, and the Ewald parameter $\kappa$. The chosen system size in the 
non-periodic MD simulations is given in Table 2 in terms of $N$ and the radius of the droplet. In the following, we document the computational options that were generally applied throughout this study for the various types of calculations.

Periodic MD simulations. Group-based cut-offs were applied for the real-space electrostatics and the van-der-Waals (vdW) interactions (see Table 1); i.e. a water molecule was added to the cut-off list if any of its atoms was less than the cut-off distance from any QM atom. The SPME method was employed in all periodic simulations for the reciprocal summation between MM sites using a grid size of $\sim 1$ Bohr. In the Gen-Ew calculations, the QM ESP charges were considered converged when the root-mean-square deviation (rmsd) in successive iterations dropped below $1.0 \cdot 10^{-5}$, and the radius of the sphere containing the virtual charges was set equal to the cut-off for the real-space electrostatics plus $2 \AA$.

Free energy calculations. The TIP3P water molecules were kept rigid with SHAKE constraints. ${ }^{73}$ All MD simulations were performed under NVT conditions at $300 \mathrm{~K}$, the temperature was controlled with the Nosé-Hoover chain thermostat, $\frac{74-76}{-76}$ and the time step was chosen to be 1 fs. The PMF profiles for the chosen test reactions were determined using umbrella sampling. The umbrella sampling parameters adopted for the different systems are summarized in Table 3. Each umbrella sampling window was equilibrated for 10 ps followed by a production run of 40 ps (90 ps in the glycine dipeptide Ace-Gly-NMe case). The weighted histogram analysis method (WHAM $)^{77}$ was employed to determine the PMF as a function of a reaction coordinate.

Table 3: Umbrella sampling: chosen test systems, distances d used as reaction coordinate, number $M$ of sampling windows, and restraining force constants in Hartree/Bohr ${ }^{2}$.

\begin{tabular}{l|c|c|c}
\hline System & Reaction coordinate & $M$ & Force constant \\
\hline $\mathrm{NH}_{4} \mathrm{Cl}$ & $\mathrm{d}(\mathrm{N}-\mathrm{Cl})$ & 33 & 0.03 \\
$\mathrm{NH}_{4} \mathrm{PO}_{3}$ & $\mathrm{~d}(\mathrm{~N}-\mathrm{P})$ & 32 & 0.03 \\
$\mathrm{~S}_{\mathrm{N}} 2$ & $\mathrm{~d}(\mathrm{C}-\mathrm{Cl})-\mathrm{d}\left(\mathrm{C}-\mathrm{Cl}{ }^{\prime}\right)$ & 42 & 0.05 \\
allylvinyl ether & $\mathrm{d}(\mathrm{C}-\mathrm{C})-\mathrm{d}(\mathrm{C}-\mathrm{O})$ & 45 & 0.30 \\
Ace-Gly-NMe & dihedral angle $\varphi$ & 36 & 0.05 \\
\hline
\end{tabular}

Geometry optimizations. The Claisen rearrangement from chorismate to prephenate in the chorismate mutase enzyme was studied by QM/MM potential energy scans. The QM region in- 
cluded only the reacting molecule and consisted of 24 atoms with a net overall charge of - 2 . Geometry optimizations were performed using hybrid delocalized internal coordinates. ${ }^{78}$ The optimized region comprised all amino acid residues and water molecules containing atoms within a distance

of $6 \AA$ from any atom of the QM part; the coordinates of more distant protein residues and solvent molecules were kept fixed. The QM-MM electrostatic interactions were treated by electrostatic embedding. 79

Non-periodic calculations. Non-periodic QM/MM calculations were performed using the droplet model. The solute was first solvated in a water box and equilibrated by the means of 500 ps molecular dynamics in the NPT ensemble. Thereafter the system was truncated to generate a droplet model with the solute solvated in the water ball. In the non-periodic MD simulations, we either used spherical boundary conditions or simply froze the outer solvent layer to prevent evaporation. In case of spherical boundary conditions, we chose a central atom (one of the solute atoms) and applied a harmonic restraint that exerts a force acting towards the central atom on every atom farther away than some distance $\mathrm{x}_{0}$ from the central atom. This distance $\mathrm{x}_{0}$ was chosen such that the water ball keeps the proper volume. No cut-off for QM-MM electrostatic interactions was applied in the droplet model calculations.

\section{Results}

\subsection{Validation of the Gen-Ew method}

One key feature of the Gen-Ew approach is that it represents the PBC potential by virtual sphere charges and the QM density by ESP charges. These approximations cause deviations between the results from Gen-Ew and QM/MM-Ewald calculations, which will depend on the chosen number of virtual sphere charges. This is illustrated in Figure 2 for the gradient components at the QM atoms of the $\mathrm{NH}_{4} \mathrm{Cl}$ /water test system. 

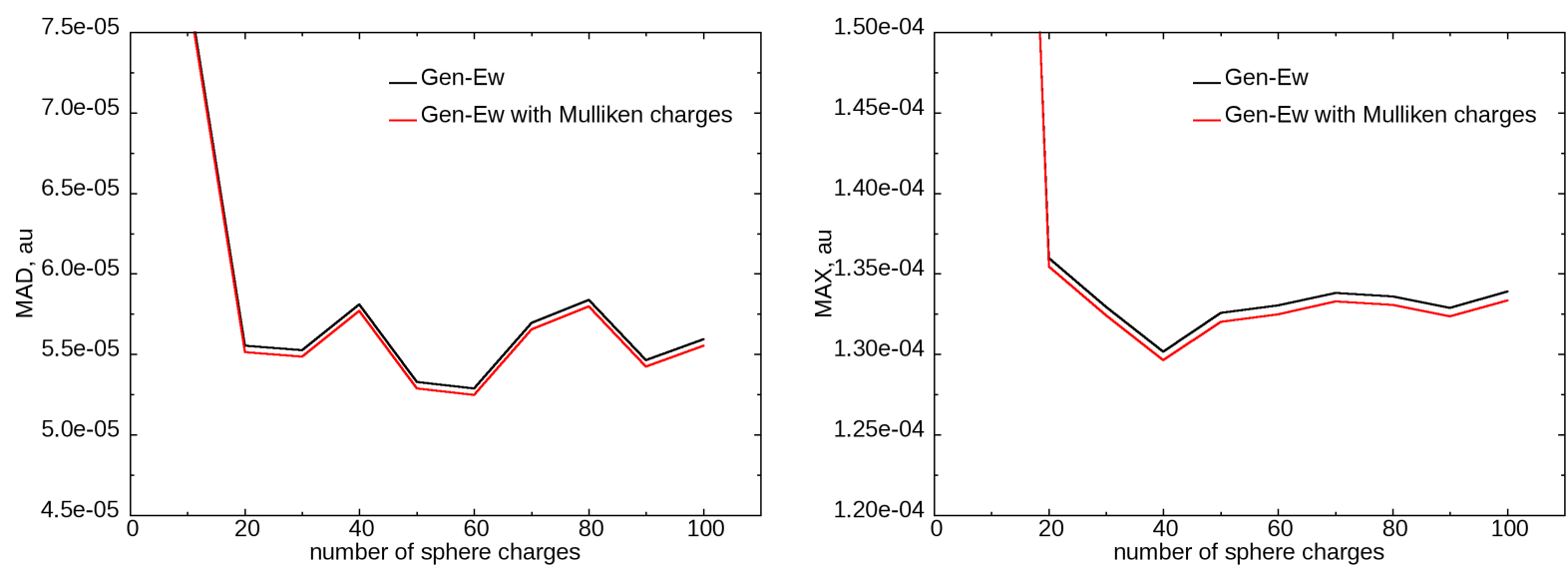

Figure 2: Mean absolute deviations (MAD, left) and maximum absolute deviations (MAX, right) between Gen-Ew and QM/MM-Ewald results: gradient components at the QM atoms of the $\mathrm{NH}_{4} \mathrm{Cl} /$ water test system.

The mean absolute deviations (MAD) and maximum absolute deviations (MAX) of the GenEw QM gradient components from the QM/MM-Ewald reference values are around $5.5 \cdot 10^{-5}$ and $1.3 \cdot 10^{-4}$ au, respectively, for 20 or more virtual sphere charges. Such deviations are not critical for QM/MM geometry optimizations, because the normally chosen convergence criterion for the maximum gradient component is considerably higher (typically $4.5 \cdot 10^{-4} \mathrm{au}$ ). In this sense, the QM/MM gradient calculation in the Gen-Ew approach can be considered converged already for a rather small number of virtual sphere charges (default choice: 80 ).

Table 4: Comparison between QM/MM-Ewald and Gen-Ew results for three test systems: MAX and MAD deviations in the gradient components (see text), deviation in the total energy $\Delta E$, and computation times.

\begin{tabular}{l|c|c|c|c|c}
\hline \multirow{2}{*}{ System } & \multirow{2}{*}{ MAX, au } & \multirow{2}{*}{ MAD, au } & \multirow{2}{*}{$\Delta E$, au } & \multicolumn{2}{|c}{ single-point cpu time, s } \\
\cline { 5 - 6 } & & & & QM/MM-Ewald & Gen-Ew \\
\hline $\mathrm{NH}_{4} \mathrm{Cl}$ in water & $1.3 \cdot 10^{-4}$ & $5.6 \cdot 10^{-5}$ & $<1 \cdot 10^{-5}$ & 5.3 & 6.2 \\
allylvinyl ether in water & $9.0 \cdot 10^{-5}$ & $3.9 \cdot 10^{-5}$ & $7 \cdot 10^{-5}$ & 3.1 & 3.8 \\
chorismate mutase & $2.3 \cdot 10^{-4}$ & $7.6 \cdot 10^{-5}$ & $2.6 \cdot 10^{-4}$ & 215 & 222 \\
\hline
\end{tabular}

Further numerical comparisons between QM/MM-Ewald and Gen-Ew results are collected in Table 4. Evidently, the deviations are of similar magnitude for all three test systems, typically less than $10^{-4}$ au for the MAD value (gradient) and of the order of $10^{-4}$ au for the total energy. The computation time for a single-point energy and gradient calculation is slightly higher for Gen-Ew 
compared with QM/MM-Ewald (Table 4). This is because the Gen-Ew method is by construction slower since it requires several QM computations (usually 2-3) and virtual charge evaluations to converge the QM ESP charges.

We also checked the importance of the contributions from ESP charge derivatives, $\frac{\partial Q_{\alpha}}{\partial \mathbf{R}_{A}}$, in the gradient calculations for the Gen-Ew approach (eqs. 9410). Without inclusion of these contributions we observed maximum absolute deviations and mean absolute deviations of the QM gradient of the order $10^{-5}$ au compared with finite-difference reference values (Table 5). The contributions from the ESP charge derivatives are thus not zero but small enough to obtain sufficiently accurate QM gradients without calculating them.

Table 5: Comparison between Gen-Ew results with and without contributions from ESP charge derivatives for two test systems: MAX and MAD deviations in the gradient components (see text).

\begin{tabular}{l|c|c}
\hline System & MAX, au & MAD, au \\
\hline $\mathrm{NH}_{4} \mathrm{Cl}$ in water & $7.9 \cdot 10^{-5}$ & $2.5 \cdot 10^{-5}$ \\
allylvinyl ether in water & $4.9 \cdot 10^{-5}$ & $1.1 \cdot 10^{-5}$ \\
\hline
\end{tabular}

We now address the origin of the deviations between the QM/MM-Ewald and Gen-Ew results. As already described above, the PBC contributions are handled in a formally different manner in these approaches, and the PBC potential is mimicked by the virtual charges only approximately (with high but finite accuracy). The second distinction is that the QM density is represented by Mulliken charges in the QM/MM-Ewald method and by ESP charges in the Gen-Ew method. These differences are illustrated for the $\mathrm{NH}_{4} \mathrm{Cl} /$ water test system in Table 6 and Figure 3. They are fairly small but non-negligible; for example, during the MD simulation the ESP charges of the $\mathrm{Cl}$ atom are less negative than the Mulliken charges (average values: -0.834 vs. -0.891 ), and they fluctuate somewhat more (standard deviations: 0.034 vs. 0.029 ). 
Table 6: Average QM charges during MD simulations of the $\mathrm{NH}_{4} \mathrm{Cl} /$ water test system: QM/MMEwald (Mulliken) versus Gen-Ew (ESP).

\begin{tabular}{c|c|c}
\hline & QM/MM-Ewald & Gen-Ew \\
\hline $\mathrm{N}$ & -0.079 & 0.172 \\
$\mathrm{H}$ & 0.252 & 0.212 \\
$\mathrm{H}$ & 0.265 & 0.170 \\
$\mathrm{H}$ & 0.275 & 0.196 \\
$\mathrm{H}$ & 0.217 & 0.163 \\
$\mathrm{Cl}$ & -0.930 & -0.913 \\
\hline
\end{tabular}

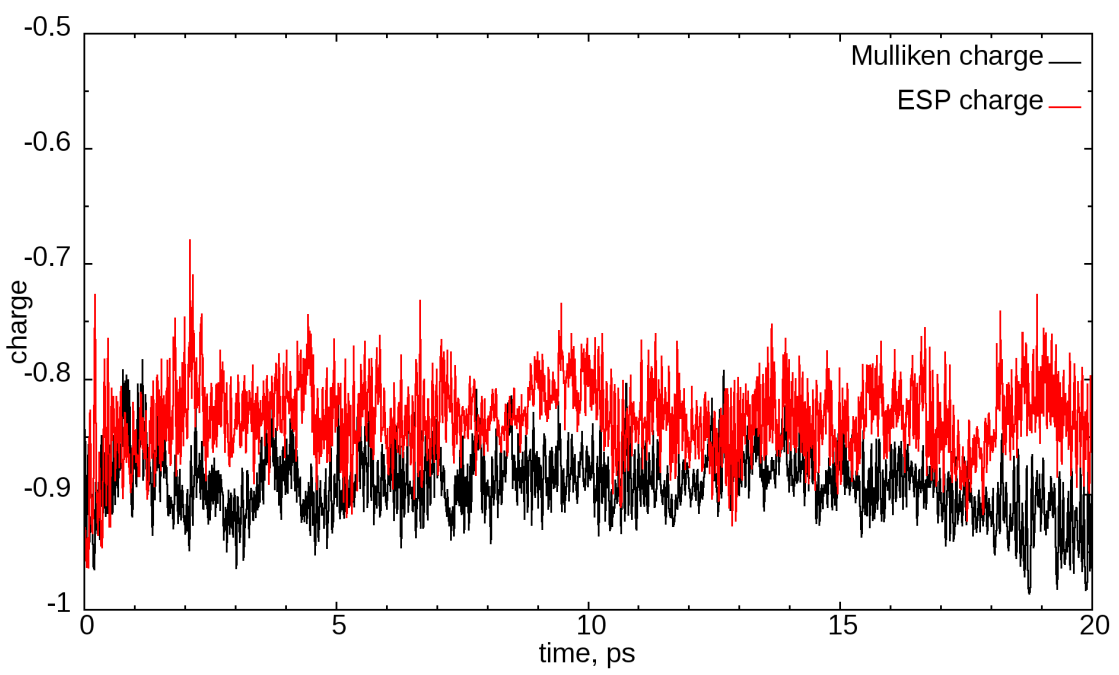

Figure 3: $\mathrm{QM}$ charge of the $\mathrm{Cl}$ atom during $\mathrm{MD}$ simulations of the $\mathrm{NH}_{4} \mathrm{Cl} /$ water test system: QM/MM-Ewald (Mulliken, black curve) versus Gen-Ew (ESP, red curve).

Finally, we examine energy conservation during MD simulations in the NVE ensemble. We performed QM-only (PM3) simulations of a single glycine molecule in the gas phase as well as non-periodic and periodic QM/MM (PM3/TIP3P) simulations of the glycine/water test system; in the non-periodic QM/MM MD simulations the outer water layer was frozen such that the same number of atoms were free to move in the periodic and non-periodic calculations. In all cases, standard SCF convergence thresholds were adopted for the energy $\left(10^{-8} \mathrm{eV}\right)$ and for the diagonal elements of the density matrix $\left(10^{-8}\right)$. In the QM-only MD simulations with a time step of $1 \mathrm{fs}$, the total energy remained constant and fluctuated within $0.5 \mathrm{kcal} / \mathrm{mol}$ (Figure 4); upon decreasing the time step in the QM-only MD simulations from 1 to $0.1 \mathrm{fs}$, the root-mean square fluctuation in 
the energy dropped from 0.074 to $0.0009 \mathrm{kcal} / \mathrm{mol}$ (data not shown). The same behavior was found in the non-periodic QM/MM MD simulations of the glycine/water test system with a time step of $1 \mathrm{fs}$ using the droplet model (Figure 5). By contrast, the total energy was not conserved in the periodic QM/MM MD simulations, neither for the QM/MM-Ewald nor for the Gen-Ew method: in both cases, there was a change of about $10 \mathrm{kcal} / \mathrm{mol}$ within $100 \mathrm{ps}$ (Figure 6). This may happen because both methods use a cut-off scheme in the PBC treatment by construction (minimum image convention): in the inner region up to a cut-off $\left(r_{c u t}\right.$, see Table 1) the MM point charges interact with the QM density directly, while outside this region the MM point charges interact with Mulliken (QM/MM-Ewald) or ESP (Gen-Ew) charges representing the QM density. This will create problems when MM molecules cross the cut-off border between the two regions during MD simulations, leading to an inherent slight discontinuity of the computed energy, which will in turn cause some slight discontinuity in the computed gradient. In view of these issues, we performed all following QM/MM-Ewald and Gen-Ew MD simulations in the NVT ensemble (section 5.2). We also note that such problems do not arise in QM/MM PBC geometry optimizations with a frozen outer region (i.e. with the optimization restricted to the relevant part of the inner region, as often done in practice).

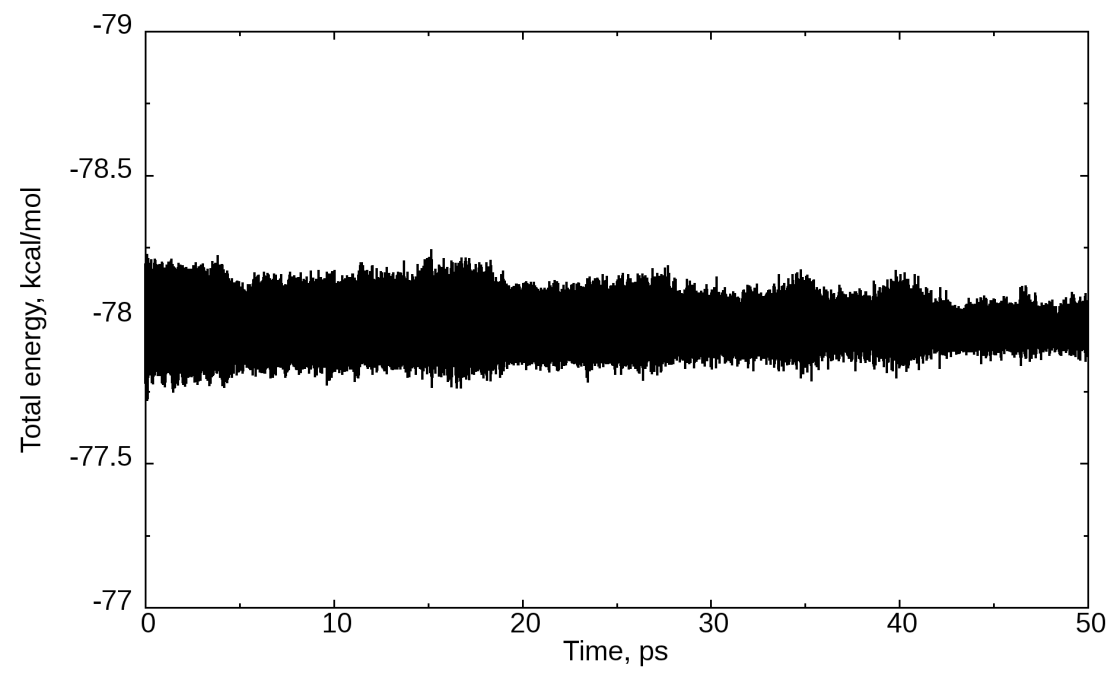

Figure 4: QM-only MD simulations in the NVE ensemble: total energy versus time for one glycine molecule (see text). 


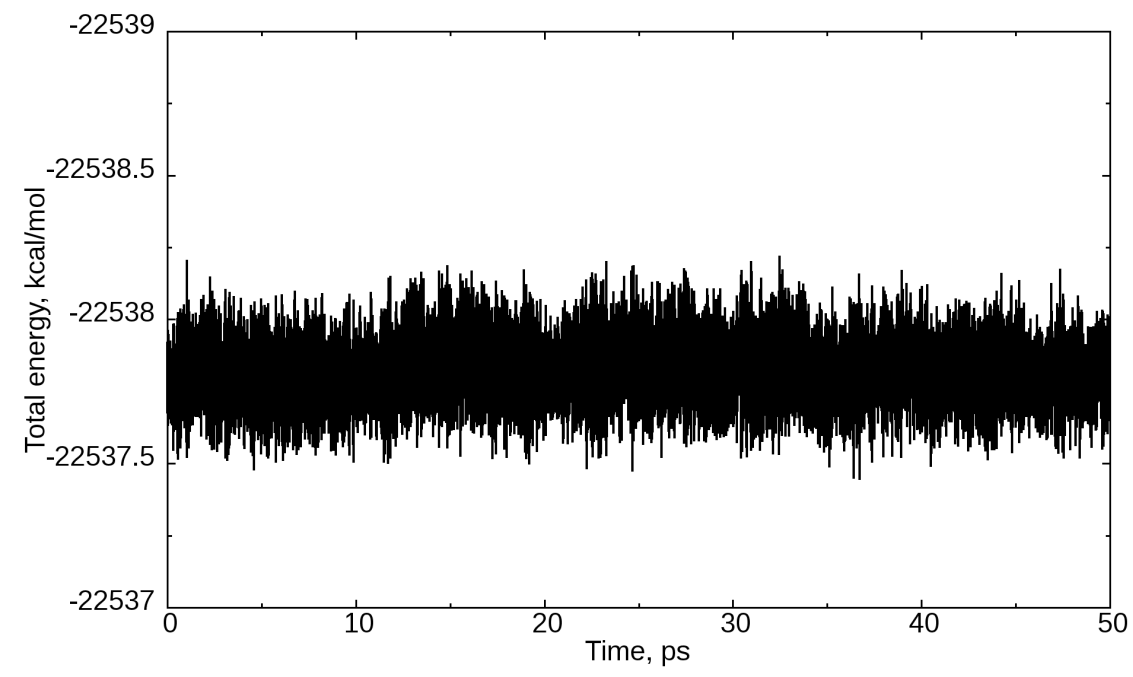

Figure 5: Non-periodic QM/MM MD simulations in the NVE ensemble using the droplet model: total energy versus time for the glycine/water test system.

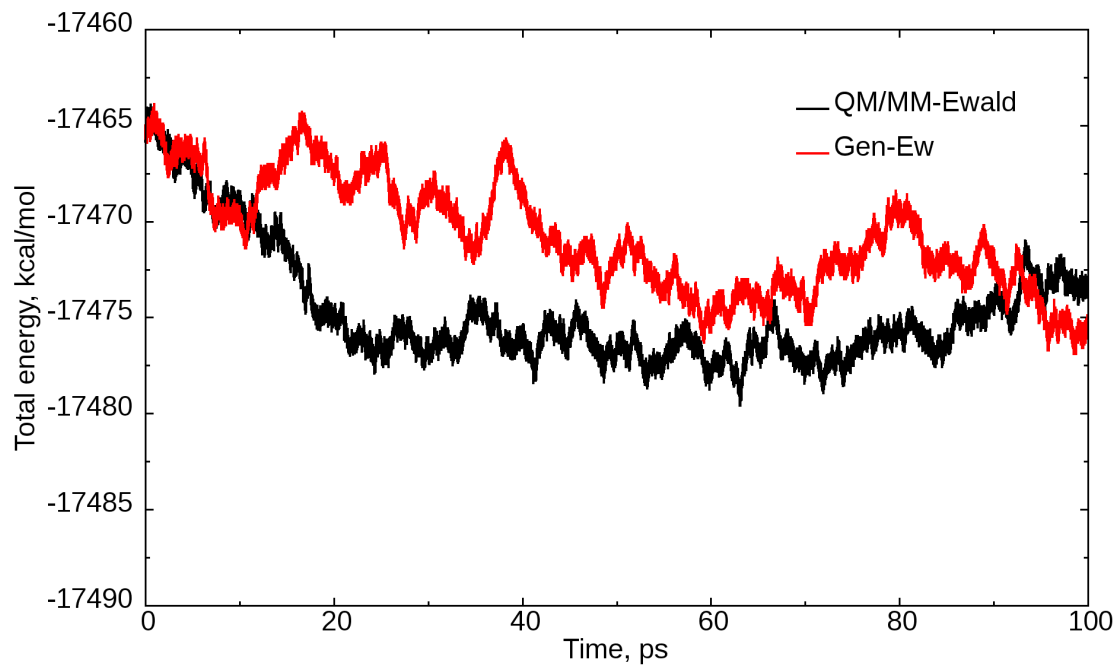

Figure 6: Periodic MD simulations in the NVE ensemble using the QM/MM-Ewald and Gen-Ew methods: total energy versus time for the glycine/water test system.

\subsection{Free energy calculations}

\subsubsection{Dissociation of salts}

Ammonium chloride. PMF profiles for $\mathrm{NH}_{4} \mathrm{Cl}$ dissociation in water were determined from simulations with periodic boundary conditions using the standard QM/MM-Ewald and the Gen-Ew 
methods and from non-periodic calculations with spherical boundary conditions (SBC); in the latter case, the nitrogen atom of the solute served as central SBC atom.

The computed PMF profiles are shown in Figure 7. They are qualitatively similar with free energy barriers of 5.5-6.0 kcal/mol from the periodic calculations and $5.5 \mathrm{kcal} / \mathrm{mol}$ from the nonperiodic calculations with a $20 \AA$ solvent sphere; they all become rather flat beyond an $\mathrm{N}-\mathrm{Cl}$ separation of $6 \AA$. The three PMF profiles in Figure 7 generally agree to within $1 \mathrm{kcal} / \mathrm{mol}$ over the whole range of $\mathrm{N}-\mathrm{Cl}$ distances and have the same shape.

The same system was studied previously at the AM1/TIP3P level using the standard QM/MMEwald method. 18 The previously reported $\mathrm{PMF}$ curve for $\mathrm{NH}_{4} \mathrm{Cl}$ dissociation is similar to the one obtained presently but has a slightly smaller free energy barrier of $3.5 \mathrm{kcal} / \mathrm{mol} .18$ These small deviations reflect small differences in the chosen computational protocols, for example concerning the use of different vdW parameters for QM/MM interactions and of different MD ensembles (NVT vs. NPT).

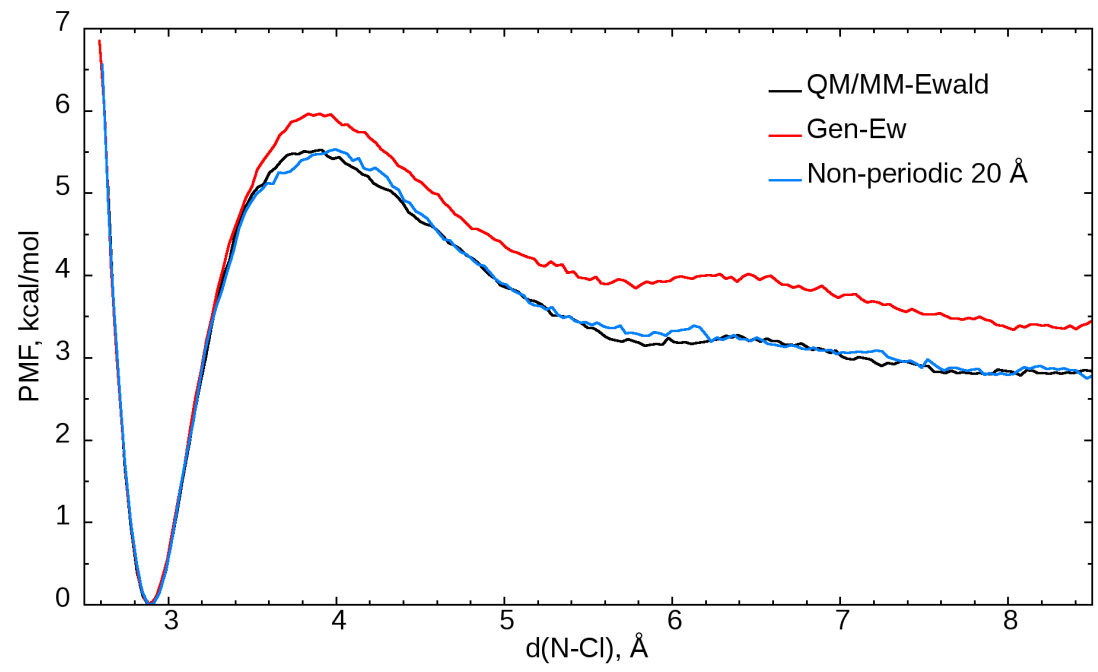

Figure 7: Potential of mean force profiles for ammonium chloride dissociation in water from AM1/TIP3P calculations: periodic and non-periodic simulations.

We also examined the question of how large the solvent shell should be in non-periodic calculations to properly capture all QM/MM electrostatic interactions. For this purpose, we performed two additional non-periodic simulations in water spheres with radii of 12 and $15 \AA$ (Figure 8). 


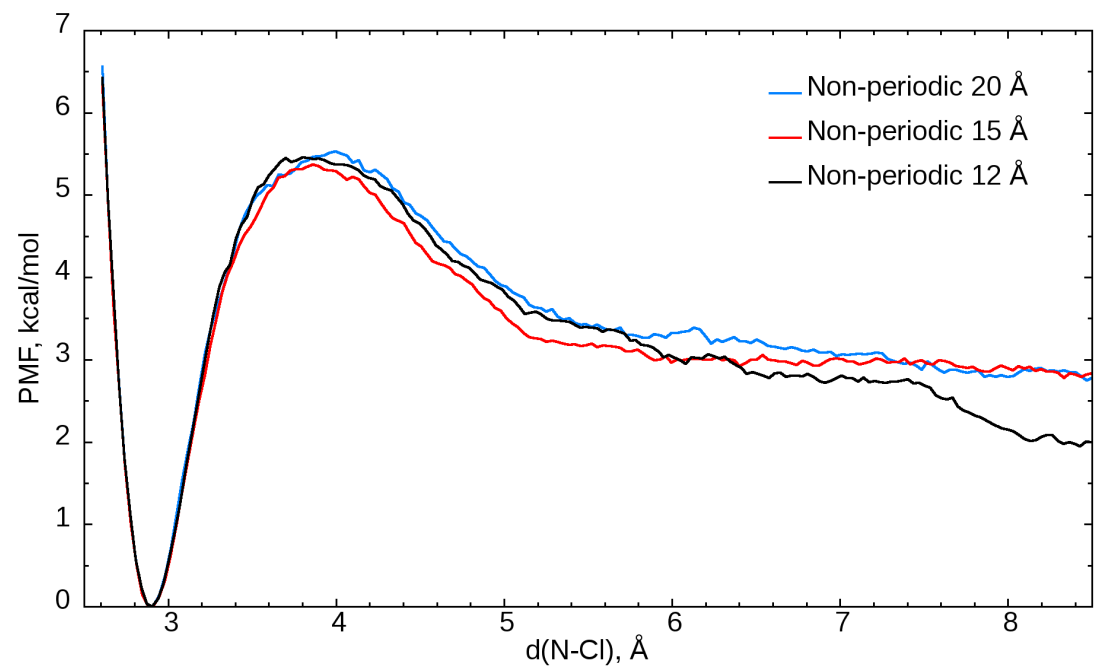

Figure 8: Potential of mean force profiles for ammonium chloride dissociation in water from AM1/TIP3P calculations: non-periodic simulations with water spheres of different size.

The PMF profiles from the non-periodic simulations with water spheres of 20,15 , and $12 \AA$ are generally similar, especially at small $\mathrm{N}-\mathrm{Cl}$ distances. However, in the case of the $12 \AA$ system, a deviating behavior is observed beyond an $\mathrm{N}-\mathrm{Cl}$ separation of $7.5 \AA$, with the energy falling off too quickly (Figure 8). This is easily rationalized: in this calculation the $\mathrm{NH}_{4}{ }^{+}$fragment is located at the center of the sphere (SBC convention), and hence $\mathrm{Cl}^{-}$moves towards the surface; at an $\mathrm{N}-\mathrm{Cl}$ separation of $7.5 \AA$ the $\mathrm{Cl}^{-}$ion is only $4.5 \AA$ away from the surface, which is apparently too close for an adequate inclusion of all relevant interactions with the bulk solvent. This example serves as a reminder that a sufficiently large solvent shell is required in non-periodic QM/MM calculations for a realistic treatment of solute-solvent interactions.

Ammonium metaphosphate. The periodic and non-periodic QM/MM simulations yield essentially the same PMF profiles for dissociation of ammonium metaphosphate in water (Figure 9). These profiles are barrierless and become flat beyond an N-P separation of $6 \AA$. 


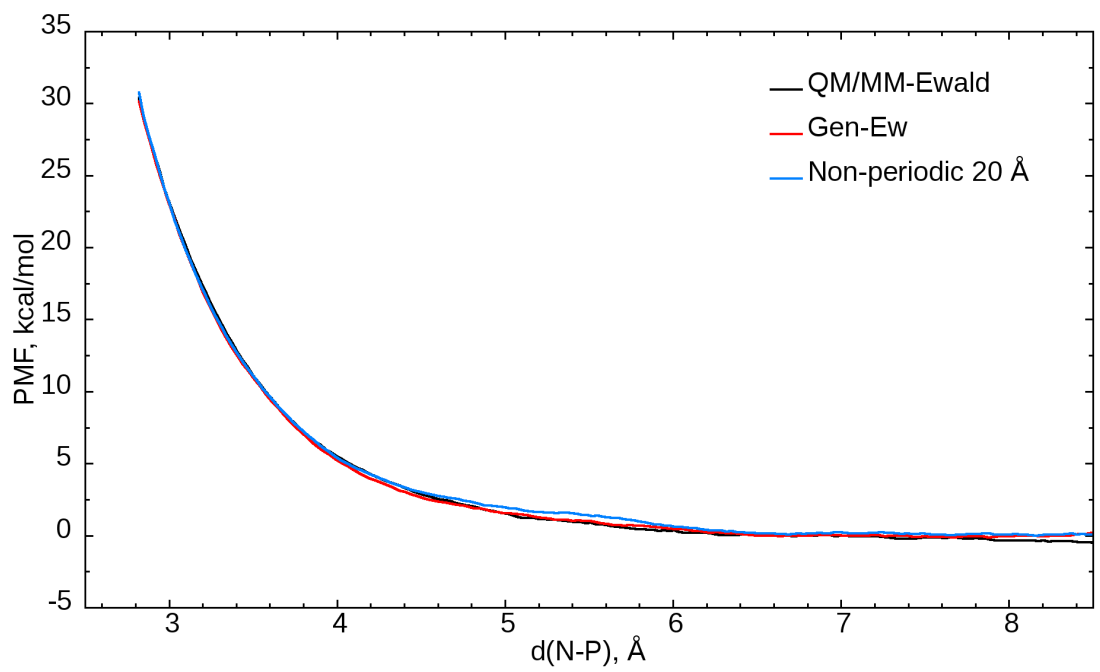

Figure 9: Potential of mean force profiles for ammonium metaphosphate dissociation in water from $\mathrm{QM}(\mathrm{MNDO} / \mathrm{d}) / \mathrm{TIP} 3 \mathrm{P}$ calculations: periodic and non-periodic simulations.

\subsubsection{Claisen rearrangement of allylvinyl ether}

The Claisen rearrangement is a $\mathrm{C}-\mathrm{C}$ bond forming pericyclic reaction (Figure 10) that is accelerated in aqueous solutions.
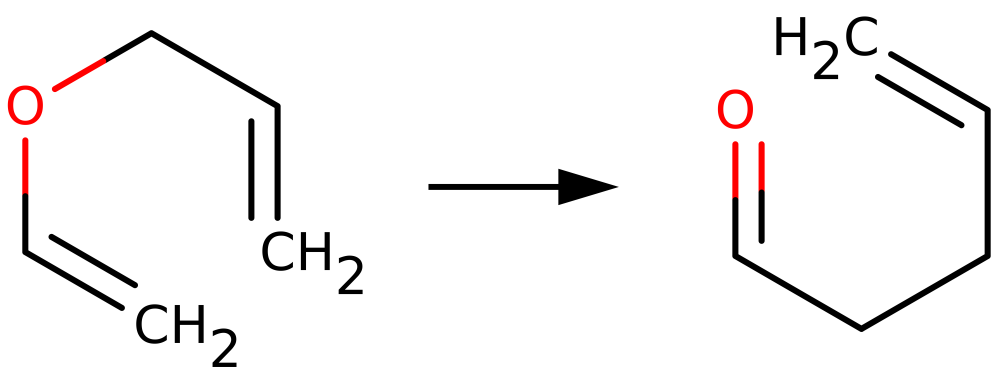

Figure 10: Claisen rearrangement of allylvinyl ether.

Two non-periodic QM/MM simulations were performed in water balls with radii of 15 and 20 $\AA$ using spherical boundary conditions, with the oxygen atom of the solute serving as the central atom (for SBC). The reaction coordinate was chosen to be the difference between the distances in the forming $\mathrm{C}-\mathrm{C}$ bond and the breaking $\mathrm{C}-\mathrm{O}$ bond. 


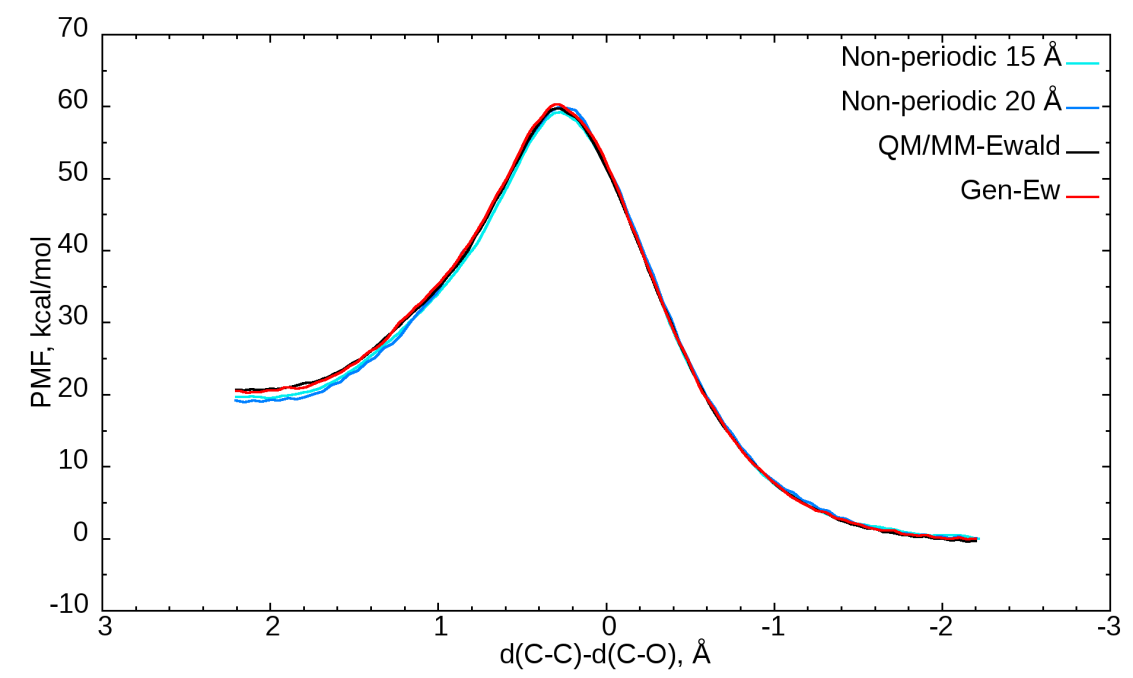

Figure 11: Potential of mean force profiles for the Claisen rearrangement of allylvinyl ether in water from PM3-PDDG/TIP3P calculations: periodic and non-periodic simulations.

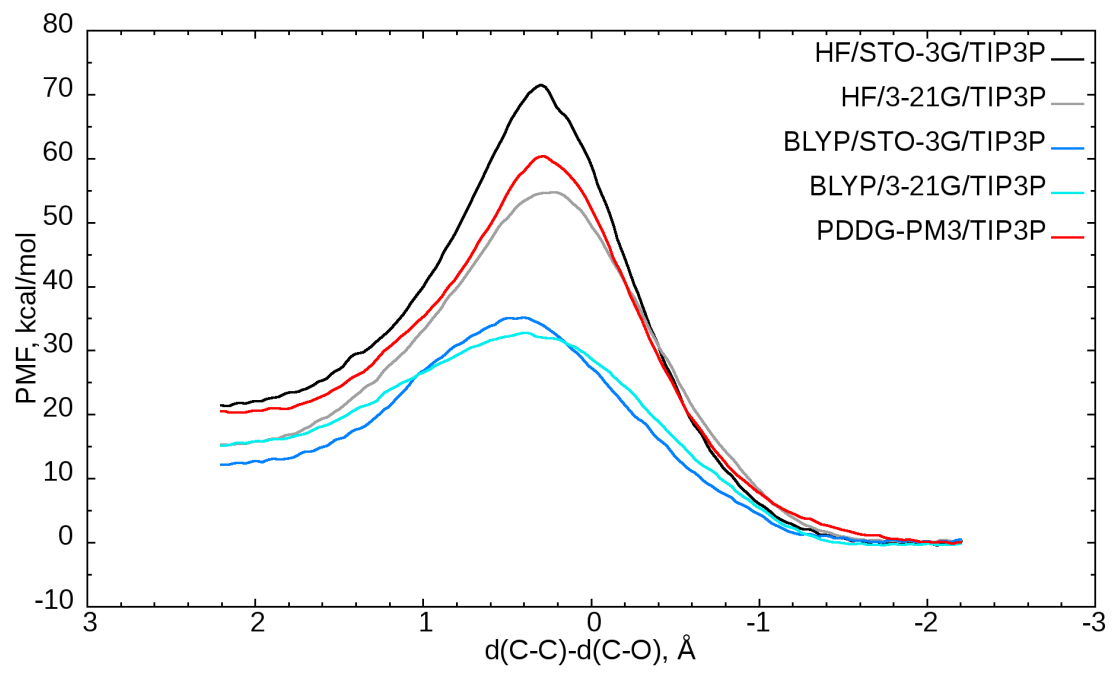

Figure 12: Potential of mean force profiles for the Claisen rearrangement of allylvinyl ether in water from periodic QM/TIP3P calculations (Gen-Ew; QM = PM3-PDDG, HF/STO-3G, HF/321G, BLYP/STO-3G, BLYP/3-21G).

The periodic and non-periodic QM/MM simulations give almost identical PMF profiles for the Claisen rearrangement of allylvinyl ether in water (Figure 11), with free energy barriers and reaction free energies of about 40 and $20 \mathrm{kcal} / \mathrm{mol}$, respectively. The four computed PMF profiles generally agree to within $1-2 \mathrm{kcal} / \mathrm{mol}$ throughout the entire reaction. In the periodic calculations, 
the QM/MM-Ewald curve is well reproduced at the Gen-Ew level, and in the non-periodic calculations, the results are apparently well converged already for the smaller water sphere (radius of 15 A). The close similarity between the PMF curves from the periodic and non-periodic simulations suggests that long-range electrostatic interactions in this reaction are well captured by the use of a finite water ball.

To illustrate that the Gen-Ew approach can be used with any QM method, we performed free energy QM/MM calculations of the Claisen rearrangement reaction using the Hartree-Fock (HF) method and density functional theory (DFT) as QM components (Figure 12). To minimize the computational effort, we applied the minimal STO-3G and the split-valence $3-21 \mathrm{G}$ basis sets. The computed free energy barriers from the HF/MM calculations bracket the PM3-PDDG/MM value while those from the DFT/MM calculations are significantly lower.

\subsubsection{Chloride exchange reaction}

In the simple halide exchange $\mathrm{S}_{\mathrm{N}} 2$ reaction, $\mathrm{Cl}^{-}+\mathrm{CH}_{3} \mathrm{Cl} \rightarrow \mathrm{CH}_{3} \mathrm{Cl}^{\prime}+\mathrm{Cl}^{-}$, one may expect to see pronounced long-range electrostatic effects. Therefore we investigated this reaction using both non-periodic and periodic QM/MM free energy calculations. In the model system for the nonperiodic simulations, the outer $4 \AA$ water layer was frozen to avoid evaporation. 


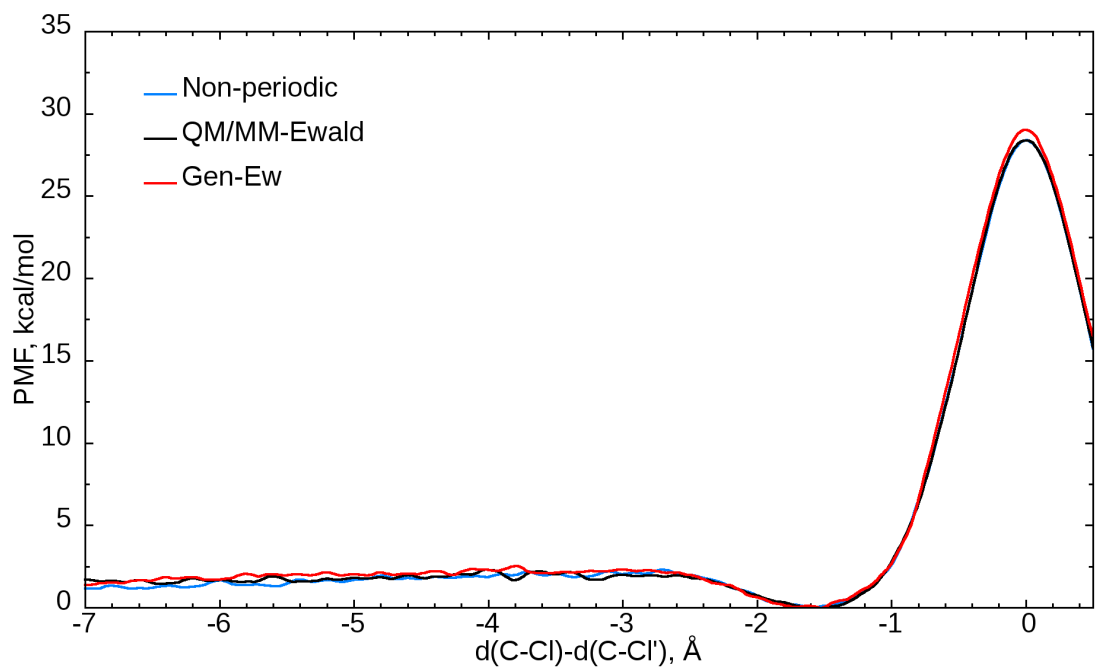

Figure 13: Potential of mean force profiles for the chloride exchange reaction in water from PM3PDDG/TIP3P calculations: periodic and non-periodic simulations.

The chloride exchange reaction is symmetric so that it is sufficient to calculate only half of the PMF profile in each case. All computed PMF curves have similar shapes: they are quite flat in the entrance and exit channels (reaction coordinate below $-3 \AA$ and above $+3 \AA$, solvent-shielded reactants and products, respectively), and there is a shallow minimum at closer distances (iondipole complex) before the PMF profiles rise sharply upon approaching the symmetric transition state (Figure 13). The free energy barriers obtained from the periodic and non-periodic simulations lie in the range of $28.5-29.0 \mathrm{kcal} / \mathrm{mol}$ and are thus close to each other. Apparently, this reaction can be described equally well by periodic and non-periodic QM/MM simulations.

\subsubsection{Glycine dipeptide rotation}

Finally, we studied the internal rotation of glycine dipeptide Ace-Gly-NMe in water (around the backbone dihedral angle $\varphi$, Figure 14) using periodic and non-periodic QM/MM simulations. Nonperiodic simulations were performed in a water ball with a radius of $20 \AA$ using spherical boundary conditions, with the $\mathrm{C} \alpha$ atom of the solute as the central atom (for SBC). 


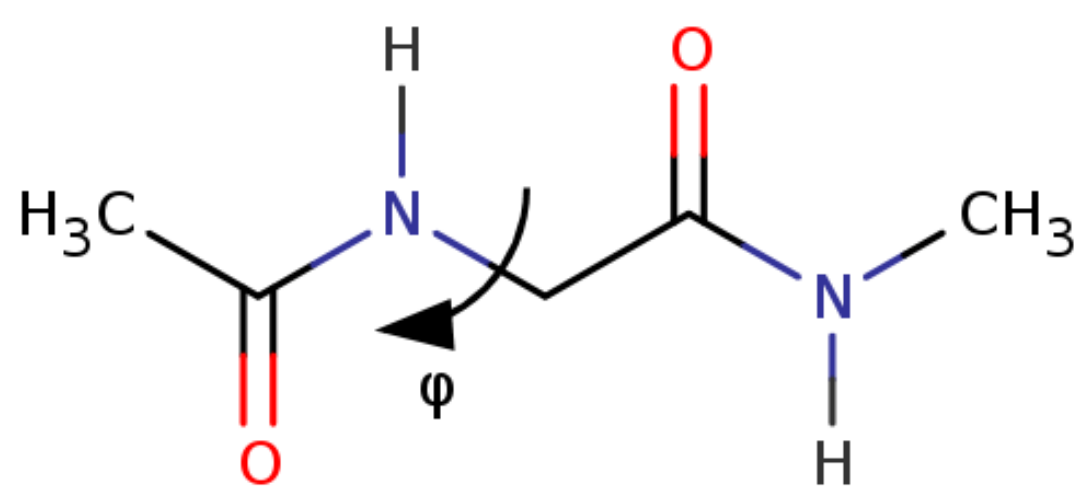

Figure 14: Schematic representation of the glycine dipeptide molecule. The backbone dihedral angle $\varphi$ is shown.

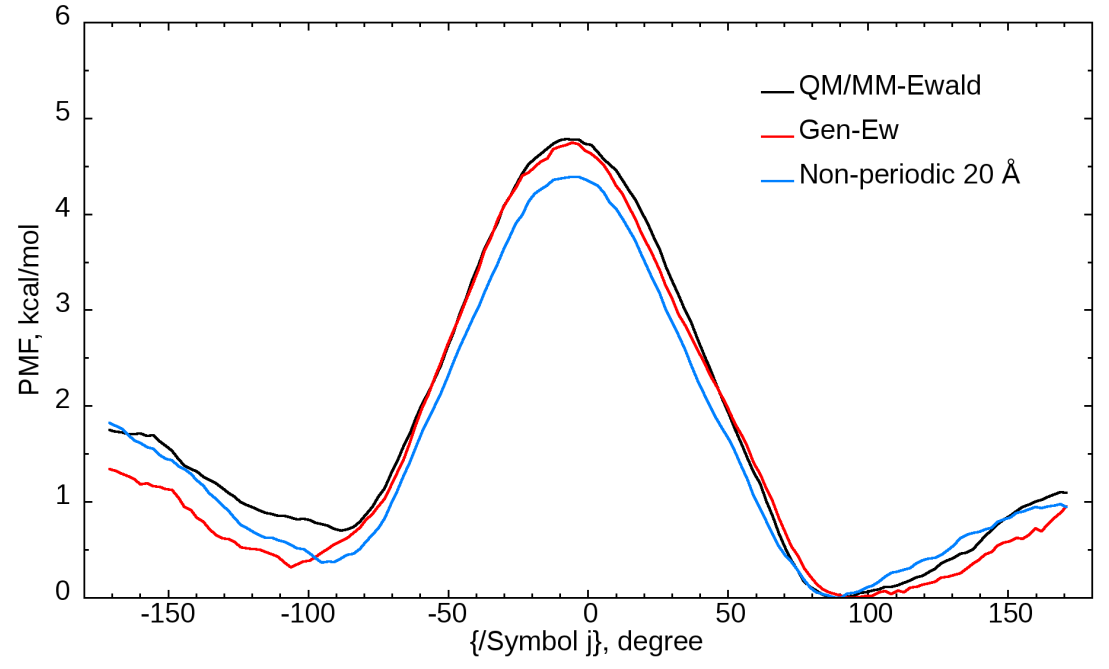

Figure 15: Potential of mean force profiles for the rotation of glycine dipeptide in water around the backbone dihedral angle $\varphi$ : periodic and non-periodic AM1/TIP3P simulations.

The computed PMF profiles from periodic and non-periodic QM/MM simulations are again quite similar over the whole range of dihedral angles, with minor deviations of less than $1 \mathrm{kcal} / \mathrm{mol}$ (Figure 15). The free energy barriers to internal rotation are calculated to be around $4.5 \mathrm{kcal} / \mathrm{mol}$ (non-periodic) and $5.0 \mathrm{kcal} / \mathrm{mol}$ (periodic). 


\subsection{Geometry optimization}

The enzyme chorismate mutase catalyzes the Claisen rearrangement of chorismate to prephenate (Figure 16). The setup for chorismate mutase was adapted from previous work of our group ${ }^{80}$ The system was solvated in a water box containing 16966 water molecules (TIP3P) and equilibrated by means of a 500 ps classical MD simulation in the NPT ensemble using the NAMD program. ${ }^{[81}$ For the non-periodic calculations it was truncated such that only water molecules within $12 \AA$ of the protein were kept. The difference between the distances in the forming $\mathrm{C}-\mathrm{C}$ bond and the breaking $\mathrm{C}-\mathrm{O}$ bond was chosen as reaction coordinate. The geometries along the reaction coordinate were optimized using the periodic QM/MM-Ewald and Gen-Gw approaches as well as a non-periodic QM/MM treatment with a $20 \AA$ water sphere.

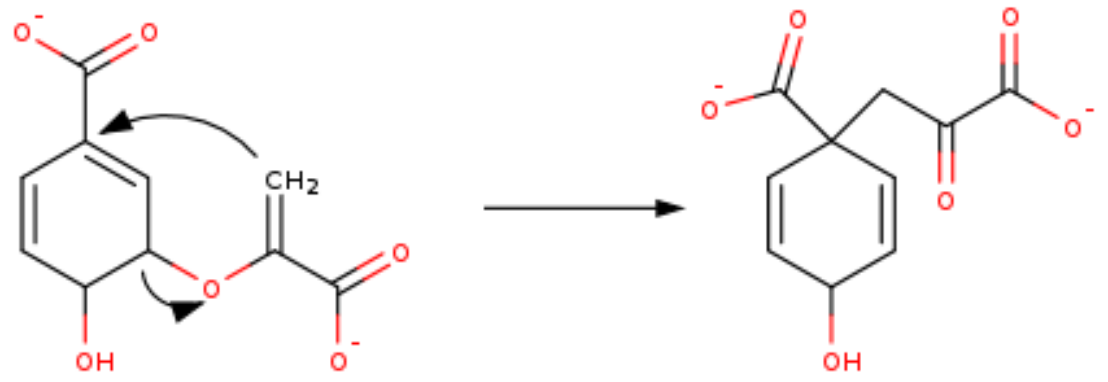

Figure 16: Claisen rearrangement from chorismate to prephenate catalyzed by chorismate mutase. 


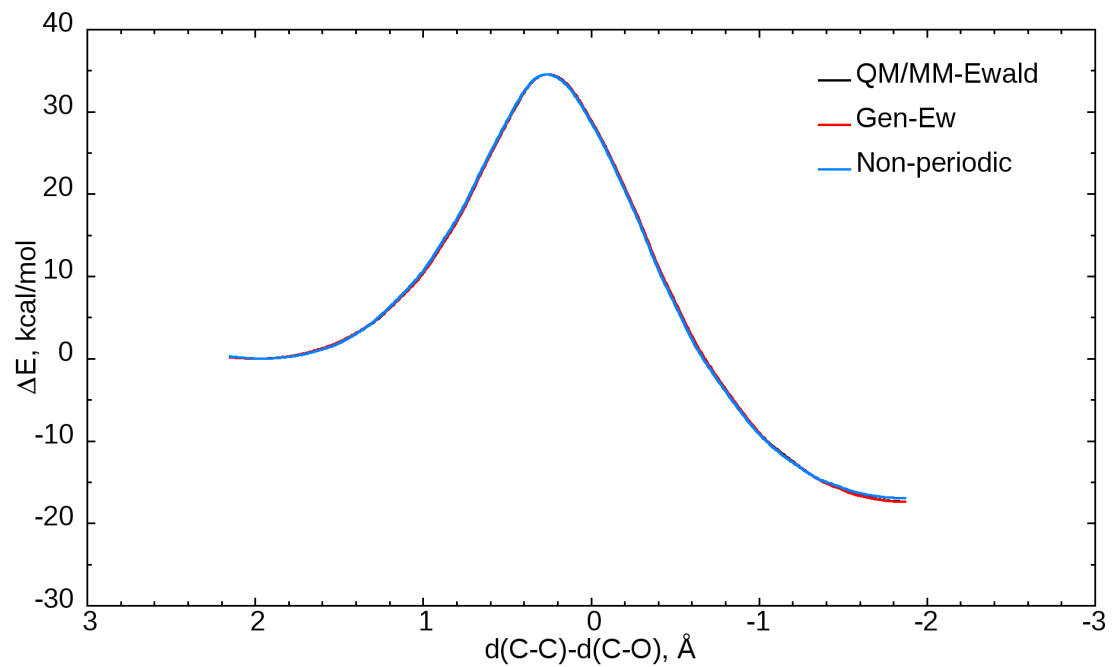

Figure 17: Potential energy profile of the Claisen rearrangement in chorismate mutase from AM1/CHARMM calculations.

The computed potential energy profiles are virtually indistinguishable (Figure 17). Evidently, the adopted periodic and non-periodic procedures capture the long-range effects of the protein environment in an essentially equivalent manner.

\section{Conclusions}

We present a general method (Gen-Ew) for periodic QM/MM calculations that can be used with any QM method, without requiring changes in the QM software. Being an approximation to the established QM/MM-Ewald method, the Gen-Ew approach represents the PBC potential by virtual sphere charges and the QM density by ESP charges. The deviations between Gen-Ew and QM/MM-Ewald results are generally small enough to justify application of the Gen-Ew method.

We report free energy calculations for five test reactions in water and potential energy scans for the Claisen rearrangement in chorismate mutase using periodic QM/MM calculations (QM/MMEwald, Gen-Ew) as well as non-periodic QM/MM calculations (droplet model). The periodic and non-periodic QM/MM treatments give similar PMF profiles for the test reactions in solution (free energy deviations of the order of $1 \mathrm{kcal} / \mathrm{mol}$ ) and essentially the same energy profile for the 
reaction in chorismate mutase. Non-periodic QM/MM calculations in a water droplet of reasonable size (radius of 15-20 $\AA$ ) thus appear to capture long-range electrostatic interactions well enough in all cases considered presently. This provides further justification for the use of the computationally efficient droplet model in QM/MM studies of reactions in solution and in enzymes.

\section{Acknowledgement}

We acknowledge support from the Cluster of Excellence RESOLV (EXC 1069) funded by the Deutsche Forschungsgemeinschaft. 


\section{References}

(1) Reichardt, C.; Welton, T. Solvents and Solvent Effects in Organic Chemistry, 4th edition; Wiley-VCH: Weinheim, 2010.

(2) Li, C.-J. Chem. Rev. 1993, 93, 2023-2035.

(3) Li, C.-J. Chem. Rev. 2005, 105, 3095-3165.

(4) Cramer, C. J.; Truhlar, G., Donald Chem. Rev. 1999, 99, 2161-2200.

(5) Tomasi, J.; Mennucci, B.; Cammi, R. Chem. Rev. 2005, 105, 2999-3094.

(6) White, W. N.; Wolfarth, E. F. J. Org. Chem. 1970, 35, 2196-2199.

(7) White, W. N.; Wolfarth, E. F. J. Org. Chem. 1970, 35, 3585-3585.

(8) Gajewski, J. J.; Jurayj, J.; Kimbrough, D. R.; Gande, M. E.; Ganem, B.; Carpenter, B. K. J. Am. Chem. Soc. 1987, 109, 1170-1186.

(9) Brandes, E.; Grieco, P. A.; Gajewski, J. J. J. Org. Chem. 1989, 54, 515-516.

(10) Rideout, D. C.; Breslow, R. J. Am. Chem. Soc. 1980, 102, 7816-7817.

(11) Breslow, R. Acc. Chem. Res. 1991, 24, 159-164.

(12) Junk, G.; Svec, H. J. Am. Chem. Soc. 1963, 85, 839-845.

(13) Ding, Y.; Krogh-Jespersen, K. Chem. Phys. Lett. 1992, 199, 262-266.

(14) Senn, H. M.; Thiel, W. Angew. Chem., Int. Ed. 2009, 48, 1198-1229.

(15) Piana, S.; Lindorff-Larsen, K.; Dirks, R. M.; Salmon, J. K.; Dror, R. O.; Shaw, D. E. PLoS One 2012, 7, e39918.

(16) York, D. M.; Yang, W.; Lee, H.; Darden, T.; Pedersen, L. G. J. Am. Chem. Soc. 1995, 117, 5001-5002. 
(17) Norberg, J.; Nilsson, L. Biophys. J. 2000, 79, 1537-1553.

(18) Nam, K.; Gao, J.; York, D. M. J. Chem. Theory Comput. 2005, 1, 2-13.

(19) Walker, R. C.; Crowley, M. F.; Case, D. A. J. Comput. Chem. 2008, 29, 1019-1031.

(20) Seabra, G. d. M.; Walker, R. C.; Elstner, M.; Case, D. A.; Roitberg, A. E. J. Phys. Chem. A 2007, $111,5655-5664$.

(21) Laino, T.; Mohamed, F.; Laio, A.; Parrinello, M. J. Chem. Theory Comput. 2006, 2, 13701378.

(22) Sanz-Navarro, C. F.; Grima, R.; García, A.; Bea, E. A.; Soba, A.; Cela, J. M.; Ordejón, P. Theor. Chem. Acc. 2010, 128, 825-833.

(23) Doll, K.; Jacob, T. J. Theor. Comput. Chem. 2015, 14, 1550054.

(24) Holden, Z. C.; Richard, R. M.; Herbert, J. M. J. Chem. Phys. 2013, 139, 244108.

(25) Holden, Z. C.; Richard, R. M.; Herbert, J. M. J. Chem. Phys. 2015, 142, 059901.

(26) Nam, K. J. Chem. Theory Comput. 2014, 10, 4175-4183.

(27) Benighaus, T.; Thiel, W. J. Chem. Theory Comput. 2009, 5, 3114-3128.

(28) Kuechler, E. R.; York, D. M. J. Chem. Phys. 2014, 140, 054109.

(29) Basilevsky, M. V.; Chudinov, G. E.; Napolov, D. V. J. Phys. Chem. 1993, 97, 3270-3277.

(30) Park, K.; Götz, A. W.; Walker, R. C.; Paesani, F. J. Chem. Theory Comput. 2012, 8, 28682877.

(31) Chandrasekhar, J.; Smith, S. F.; Jorgensen, W. L. J. Am. Chem. Soc. 1985, 107, 154-163.

(32) Cramer, C. J.; Truhlar, D. G. J. Am. Chem. Soc. 1992, 114, 8794-8799.

(33) Gao, J. J. Am. Chem. Soc. 1994, 116, 1563-1564. 
(34) Severance, D. L.; Jorgensen, W. L. J. Am. Chem. Soc. 1992, 114, 10966-10968.

(35) Jorgensen, W. L.; Blake, J. F.; Lim, D.; Severance, D. L. J. Chem. Soc., Faraday Trans. 1994, $90,1727$.

(36) Davidson, M. M.; Hillier, I. H.; Hall, R. J.; Burton, N. A. J. Am. Chem. Soc. 1994, 116, 9294-9297.

(37) Gould, I. R.; Cornell, W. D.; Hillier, I. H. J. Am. Chem. Soc. 1994, 116, 9250-9256.

(38) Iwaoka, M.; Okada, M.; Tomoda, S. J. Mol. Struct.:THEOCHEM 2002, 586, 111-124.

(39) Lyne, P. D.; Mulholland, A. J.; Richards, W. G. J. Am. Chem. Soc. 1995, 117, 11345-11350.

(40) Martí, S.; Andrés, J.; Moliner, V.; Silla, E.; Tuñón, I.; Bertrán, J. J. Phys. Chem. B 2000, 104, 11308-11315.

(41) Martí, S.; Andrés, J.; Moliner, V.; Silla, E.; Tuñón, I.; Bertrán, J. Theor. Chem. Acc. 2001, $105,207-212$.

(42) Ranaghan, K. E.; Ridder, L.; Szefczyk, B.; Sokalski, W. A.; Hermann, J. C.; Mulholland, A. J. Mol. Phys. 2003, 101, 2695-2714.

(43) Lee, Y. S.; Worthington, S. E.; Krauss, M.; Brooks, B. R. J. Phys. Chem. B 2002, 106, $12059-$ 12065 .

(44) Martí, S.; Andrés, J.; Moliner, V.; Silla, E.; Tuñón, I.; Bertrán, J.; Field, M. J. J. Am. Chem. Soc. 2001, 123, 1709-1712.

(45) Guo, H.; Cui, Q.; Lipscomb, W. N.; Karplus, M. Proc. Natl. Acad. Sci. U. S. A. 2001, 98, 9032-9037.

(46) Hur, S.; Bruice, T. C. J. Am. Chem. Soc. 2003, 125, 1472-1473. 
(47) Martí, S.; Andrés, J.; Moliner, V.; Silla, E.; Tuñón, I.; Bertrán, J. J. Mol. Struct.:THEOCHEM 2003, 632, 197-206.

(48) Martí, S.; Andrés, J.; Moliner, V.; Silla, E.; Tuñón, I.; Bertrán, J. J. Am. Chem. Soc. 2004, $126,311-319$.

(49) Martí, S.; Andrés, J.; Moliner, V.; Silla, E.; Tuñón, I.; Bertrán, J. Chem. - Eur. J. 2003, 9, 984-991.

(50) Ranaghan, K. E.; Ridder, L.; Szefczyk, B.; Sokalski, W. A.; Hermann, J. C.; Mulholland, A. J. Org. Biomol. Chem. 2004, 2, 968-980.

(51) Claeyssens, F.; Ranaghan, K. E.; Manby, F. R.; Harvey, J. N.; Mulholland, A. J. Chem. Commun. 2005, 5068.

(52) Zhang, X.; Zhang, X.; Bruice, T. C. Biochemistry 2005, 44, 10443-10448.

(53) Szefczyk, B.; Mulholland, A. J.; Ranaghan, K. E.; Sokalski, W. A. J. Am. Chem. Soc. 2004, $126,16148-16159$.

(54) Szefczyk, B.; Claeyssens, F.; Mulholland, A. J.; Sokalski, W. A. Int. J. Quantum Chem. 2007, 107, 2274-2285.

(55) Giraldo, J.; Roche, D.; Rovira, X.; Serra, J. FEBS Lett. 2006, 580, 2170-2177.

(56) Ishida, T.; Fedorov, D. G.; Kitaura, K. J. Phys. Chem. B 2006, 110, 1457-1463.

(57) Crehuet, R.; Field, M. J. J. Phys. Chem. B 2007, 111, 5708-5718.

(58) Claeyssens, F.; Ranaghan, K. E.; Lawan, N.; Macrae, S. J.; Manby, F. R.; Harvey, J. N.; Mulholland, A. J. Org. Biomol. Chem. 2011, 9, 1578-1590.

(59) Senn, H. M.; Kästner, J.; Breidung, J.; Thiel, W. Can. J. Chem. 2009, 87, 1322-1337. 
(60) Claeyssens, F.; Harvey, J. N.; Manby, F. R.; Mata, R. A.; Mulholland, A. J.; Ranaghan, K. E.; Schütz, M.; Thiel, S.; Thiel, W.; Werner, H.-J. Angew. Chem., Int. Ed. 2006, 45, 6856-6859.

(61) Dewar, M. J. S.; Zoebisch, E. G.; Healy, E. F.; Stewart, J. J. P. J. Am. Chem. Soc. 1985, 107, 3902-3909.

(62) Stewart, J. J. P. J. Comput. Chem. 1989, 10, 209-220.

(63) Thiel, W.; Voityuk, A. A. J. Phys. Chem. 1996, 100, 616-626.

(64) Repasky, M. P.; Chandrasekhar, J.; Jorgensen, W. L. J. Comput. Chem. 2002, 23, 1601-1622.

(65) Tubert-Brohman, I.; Guimarães, C. R. W.; Repasky, M. P.; Jorgensen, W. L. J. Comput. Chem. 2004, 25, 138-150.

(66) Jorgensen, W. L.; Chandrasekhar, J.; Madura, J. D.; Impey, R. W.; Klein, M. L. J. Chem. Phys. 1983, 79, 926.

(67) MacKerell, A. D. et al. J. Phys. Chem. B 1998, 102, 3586-3616.

(68) Sherwood, P. et al. J. Mol. Struct:THEOCHEM 2003, 632, 1-28.

(69) Metz, S.; Kästner, J.; Sokol, A. A.; Keal, T. W.; Sherwood, P. Wiley Interdiscip. Rev.: Comput. Mol. Sci. 2014, 4, 101-110.

(70) Essmann, U.; Perera, L.; Berkowitz, M. L.; Darden, T.; Lee, H.; Pedersen, L. G. J. Chem. Phys. 1995, 103, 8577.

(71) Thiel, W. MNDO Program, Version 6.1, Mülheim an der Ruhr, Germany, 2013.

(72) Smith, W.; Forester, T. J. Mol. Graphics 1996, 14, 136-141.

(73) Ryckaert, J.-P.; Ciccotti, G.; Berendsen, H. J. J. Comput. Phys. 1977, 23, 327-341.

(74) Nosé, S. J. Chem. Phys. 1984, 81, 511-519. 
(75) Nosé, S. Mol. Phys. 1984, 52, 255-268.

(76) Hoover, W. Phys. Rev. A 1985, 31, 1695-1697.

(77) Kumar, S.; Rosenberg, J. M.; Bouzida, D.; Swendsen, R. H.; Kollman, P. A. J. Comput. Chem. 1992, 13, 1011-1021.

(78) Billeter, S. R.; Turner, A. J.; Thiel, W. Phys. Chem. Chem. Phys. 2000, 2, 2177-2186.

(79) Bakowies, D.; Thiel, W. J. Phys. Chem. 1996, 100, 10580-10594.

(80) Benighaus, T.; Thiel, W. J. Chem. Theory Comput. 2011, 7, 238-249.

(81) Phillips, J. C.; Braun, R.; Wang, W.; Gumbart, J.; Tajkhorshid, E.; Villa, E.; Chipot, C.; Skeel, R. D.; Kalé, L.; Schulten, K. J. Comput. Chem. 2005, 26, 1781-1802. 


\section{Graphical TOC Entry}

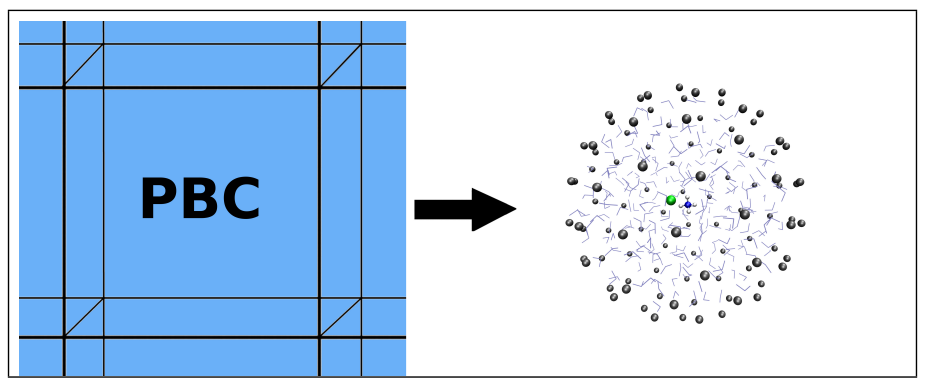

